\title{
Open Reduction and Internal Fixation of Radial Head Fractures
}

\section{Do Outcomes Differ Between Simple and Complex Injuries?}

\author{
Jeffrey M. Pike MD, MPH, Ruby Grewal MD, MSc, \\ George S. Athwal MD, Kenneth J. Faber MD, MHPE, \\ Graham J. W. King MD, MSc
}

Published online: 1 March 2014

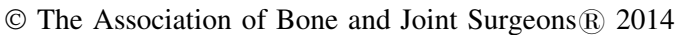

\begin{abstract}
Background Radial head fractures can occur in isolation or in association with elbow and forearm injuries. Treatment options include nonoperative management, fragment or whole-head excision, open reduction and internal fixation (ORIF), and radial head arthroplasty. However, the
\end{abstract}

One of the authors (GJWK) certifies that he has received or may receive payments or benefits, during the study period, an amount of USD 100,001 to USD 1,000,000 from Wright Medical Technologies, Inc (Arlington, TN, USA). All other authors certify that they, or a member of their immediate family, have no commercial associations (eg, consultancies, stock ownership, equity interest, patent/licensing arrangements, etc) that might pose a conflict of interest in connection with the submitted article.

All ICMJE Conflict of Interest Forms for authors and Clinical Orthopaedics and Related Research ${ }^{\mathbb{R}}$ editors and board members are on file with the publication and can be viewed on request. Clinical Orthopaedics and Related Research ${ }^{\circledR}$ neither advocates nor endorses the use of any treatment, drug, or device. Readers are encouraged to always seek additional information, including FDA approval status, of any drug or device before clinical use.

Each author certifies that his or her institution approved the human protocol for this investigation, that all investigations were conducted in conformity with ethical principles for research, and that informed consent for participation in the study was obtained.

This work was performed at the University of Western Ontario Hand and Upper Limb Centre, London, Ontario, Canada.

J. M. Pike (ه)

Department of Orthopaedic Surgery, University of British

Columbia, 590-1144 Burrard Street, Vancouver, BC V6Z 2A5,

Canada

e-mail: jeff.pike@ubc.ca

R. Grewal, G. S. Athwal, K. J. Faber, G. J. W. King

St Joseph's Health Centre, University of Western Ontario,

London, ON, Canada

G. J. W. King

e-mail: gking@uwo.ca evidence supporting ORIF for repairable radial head fractures is inconclusive.

Questions/purposes We compared patients undergoing ORIF for isolated radial head fractures or for radial head fractures associated with other fractures or elbow dislocations in terms of patient-related disability, presence of posttraumatic arthritis, complications, and rate of reoperation for capsular release.

Methods Between 1997 and 2008, 52 patients underwent ORIF of the radial head for isolated radial head fractures (simple group) and 29 underwent ORIF for radial head fracture with an associated fracture or dislocation (complex group). General indications for ORIF included displaced radial fractures, large articular surface fragments, and greater than $2 \mathrm{~mm}$ of displacement and/or a mechanical block to forearm rotation or associated fractures or ligament injuries requiring surgery. Thirty-one patients $(60 \%)$ in the simple group and 20 (69\%) in the complex group were available for followup at a mean of 4 years (range, $1.0-9.5$ years). We evaluated the patients using a validated self-reported pain and disability questionnaire (Patientrated Elbow Evaluation [PREE]). Records review included radiographic examination and assessment of major complications and secondary surgery rates for capsular release. Results With the numbers available, the groups were not different in terms of the mean PREE scores ( 8 versus 15 for the simple and complex groups, respectively; $p=0.13$, lower values indicate lower pain and disability). The simple and complex groups were also not different with the numbers available in terms of major complications (13\% versus $25 \%$, respectively; $p=0.29$ ) or secondary capsular release (3\% versus $20 \%$, respectively; $\mathrm{p}=0.07$ ).

Conclusions At short term, we found no differences between patients treated with ORIF for isolated radial head fractures and those treated for radial head fractures in 
association with other elbow injuries with regard to pain and disability scores; loss to followup in this series precludes making statements with great confidence about function after these injuries. However, the substantial capsular release and complication rates should weigh into the preoperative discussion with patients before selecting ORIF for radial head fractures.

Level of Evidence Level III, therapeutic study. See Instructions for Authors for a complete description of levels of evidence.

\section{Introduction}

Radial head fractures can occur in isolation or with associated elbow and forearm injuries, including fractures, fracturedislocations, and/or ligamentous injuries [9, 11, 32, 35]. Options for treatment include nonoperative management [1, 20], fragment or whole-head excision [6, 15, 21, 24, 30], open reduction and internal fixation (ORIF) [2, 13, 25, 26, 28], and radial head arthroplasty $[7,8,10,14,16-18]$. In addition to the particular characteristics of the radial head fracture, associated injuries may influence treatment selection.

While many options are employed in the treatment of displaced radial head fractures, the indications for ORIF of displaced radial head fractures are not clearly established $[22,23,33]$. In contrast, for irreparable fractures, there is increasing support for radial head arthroplasty as an effective treatment option $[5,16]$.

We evaluated patient-reported pain and disability in a cohort of patients with radial head fractures treated with ORIF, comparing simple radial head fractures to those with associated ligamentous or additional fracture patterns, using a validated measure. We also evaluated for the presence of posttraumatic arthritis, complication rate, and rate of reoperation for capsular release.

\section{Patients and Methods}

This study was a retrospective comparative study identifying patients with closed fractures of the radial head and neck managed with ORIF by four fellowship-trained upperextremity surgeons at a tertiary referral center between November 1997 and June 2008. A search of the hospital's electronic medical records and surgical database identified all patients treated with ORIF for radial head fractures. Institutional review board approval was obtained before initiation of the study. Exclusion criteria were skeletal immaturity $(\mathrm{n}=0)$ and those for whom initial radiographs were unavailable $(n=4)$.

Patients with a radial head fracture managed with ORIF treated within 14 days of injury were included. Indications for ORIF included displaced radial fractures with articular segments of greater than $30 \%$ of the articular surface and greater than $2 \mathrm{~mm}$ of displacement and/or a mechanical block to forearm rotation or associated fractures or ligament injuries requiring surgery. If stable fixation could not be achieved for complete articular fractures as deemed by the surgeon, a radial head arthroplasty was performed. Fractures with less articular involvement and less displacement were treated nonoperatively, including those as part of complex elbow injury. Eighty-one patients with radial head fractures treated with ORIF between November 1997 and June 2008 were identified.

Eligible patients were contacted by mail and then followed up by telephone inviting them to participate in the study. Of the 81 potential patients, two declined to participate and 28 could not be located, which left 51 patients (63\%) returning for a comprehensive evaluation. The cohort was divided into two groups based on preoperative imaging: isolated radial head fractures (simple group) (Fig. 1) and those with associated fractures or dislocations (complex group) (Fig. 2). Of the 52 potential subjects in the simple group, $31(60 \%)$ were available for followup, and of the 29 potential subjects in the complex group, 20 (69\%) were available for followup. The complex group included terrible triad fracture-dislocation variants $(\mathrm{n}=13)$, combined proximal ulna and radial head fractures $(\mathrm{n}=5)$, and radial head plus capitellum fractures $(n=2)$. The mean age of the simple group (40 years; SD, 11 years; range, 23-62 years) was 10 years younger than the complex group (50 years; $\mathrm{SD}, 10$ years; range, 22-71) $(\mathrm{p}=0.002)($ Table 1$)$. As expected, higher-grade Broberg-Morrey fracture patterns were observed in the complex group than in the simple group $(\mathrm{p}=0.004)$, with higher associated energy mechanisms $(\mathrm{p}=$ 0.07). Otherwise, no differences were observed between groups. Mean followup was 4.4 years (SD, 2.3 years; range, $1.0-9.5$ years) for the simple group and 4.3 years (SD, 2.5 years; range, 1.2-11.3 years) for the complex group.

We recorded patient demographic data (age, sex, BMI), general health measures (SF-12 mental and physical component summary scores), depression scores, occupational and recreational demands, and fracture characteristics. The Center for Epidemiologic Studies Depression Scale was used to measure depression; a score of 16 or greater suggests depression with a range in scores of 0 to 60 [31]. Occupational and recreational demands were classified based on author opinion in the absence of validated classification schemes. Occupational demands were divided into nonheavy labor and heavy labor (lifting $>15 \mathrm{~kg}$ ). Recreational demands were divided into nonweightbearing sports and weightbearing sports/contact sports. The mechanisms of injury involved in the radial head fracture were divided into low energy and high energy (falls from a height greater than 2 feet $[61 \mathrm{~cm}]$, fall during a sporting activity, or motor vehicle collision). 

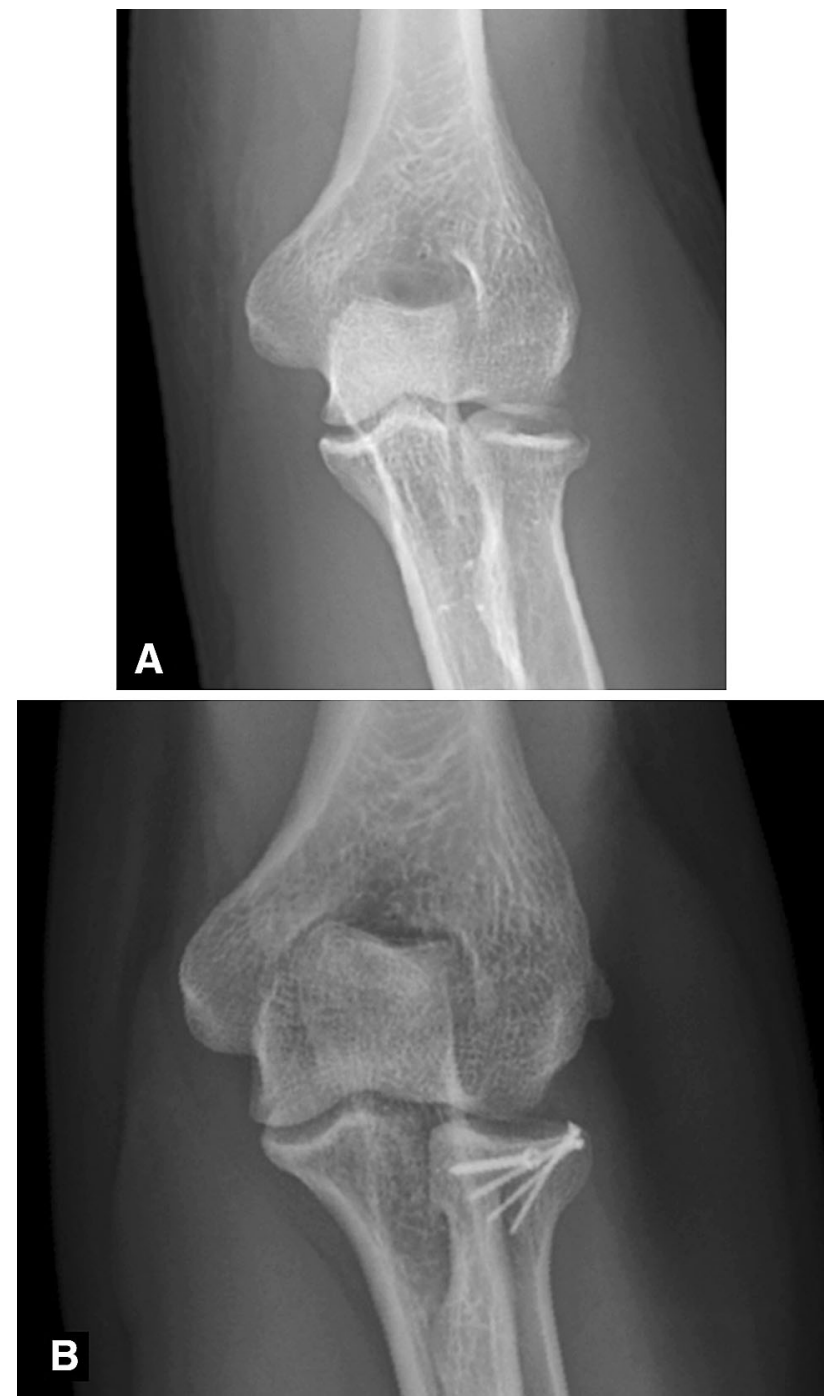

Fig. 1A-B (A) A preoperative radiograph shows an isolated radial head fracture. (B) A 5-year postoperative radiograph shows the healed fracture.

The proportion of joint surface involved and amount of displacement were estimated from the initial radiographs. CT was used at the discretion of the treating surgeon to better characterize selected fractures. Isolated radial head fractures were identified when preoperative imaging did not reveal any associated fractures or malalignment to suggest ligamentous injury. Terrible triad fracture-dislocations were defined as the combination of a radial head fracture, coronoid fracture, and posterior dislocation. To be classified as a posterior dislocation, imaging confirmed the dislocation, an impaction fracture on the posterior capitellum was identified [29] or intraoperative observation confirmed the same. Combined proximal ulna and radial head fractures were defined on imaging. Ligamentous injuries were recorded from the intraoperative findings.
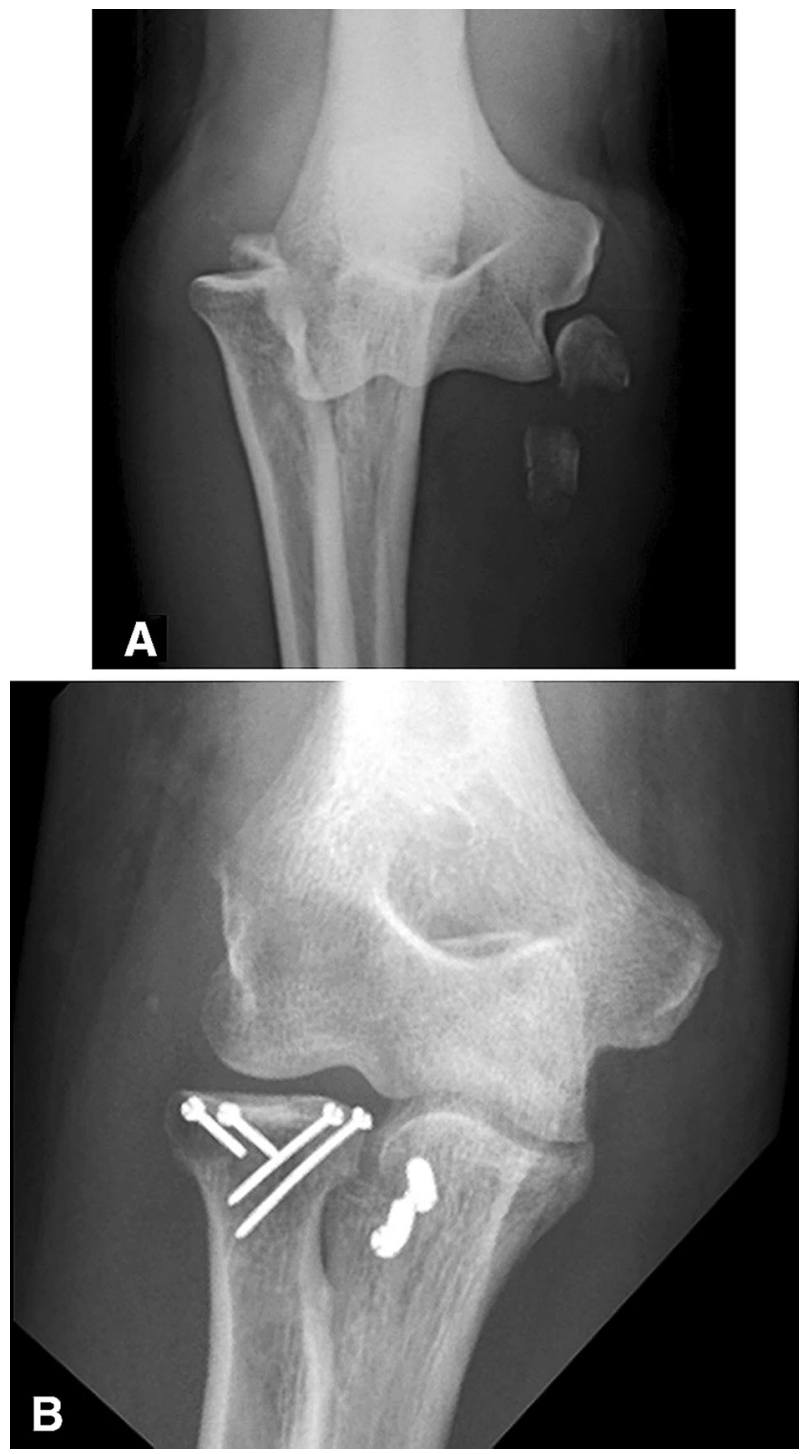

Fig. 2A-B (A) A preoperative radiograph shows a terrible triad fracture-dislocation, with a comminuted radial head fracture, coronoid fracture, and associated posterior dislocation. (B) A 2-month postoperative radiograph shows the healed fracture-dislocation.

Fractures were classified according to the Broberg and Morrey modification of the Mason classification [4]: Grade I, fractures displaced less than $2 \mathrm{~mm}$; Grade II, fractures displaced more than $2 \mathrm{~mm}$ and involving more than $30 \%$ of the head; Grade III, comminuted fractures; and Grade IV, fractures associated with dislocation. A fellowship-trained orthopaedic surgeon (JMP) who was not involved in their initial care reviewed radiographs of the affected and unaffected elbow and then subsequently examined the patients; a research assistant (CY) completed objective and subjective outcomes instruments.

All radial head fractures were exposed using a posterior skin incision and a lateral fasciocutaneous flap. A medial flap 
Table 1. Group characteristics

\begin{tabular}{lll}
\hline Variable & $\begin{array}{l}\text { Simple group (isolated } \\
\text { radial head fractures) } \\
(\mathrm{n}=31)\end{array}$ & $\begin{array}{l}\text { Complex group (radial head } \\
\text { fractures with associated } \\
\text { complex injury patterns) }(\mathrm{n}=20)\end{array}$ \\
\hline Age (years)* & $40(11)$ & $50(9)$ \\
Sex (number of females:males) & $14: 17$ & $10: 10$ \\
BMI* & $28(4)$ & $28(7)$ \\
SF-12 mental component summary (points)* & $56(7)$ & $55(9)$ \\
SF-12 physical component summary (points)* & $46(7)$ & $45(10)$ \\
CESD (points)* & $0.5(2.0)$ & $0.85(1.9)$ \\
Heavy-labor occupation $(\%)^{\dagger}$ & 55 & 55 \\
Weightbearing and/or contact sports $(\%)^{*}$ & 68 & 65 \\
Complete articular fractures $(\%)$ & 13 & 20 \\
Three or more fragments $(\%)$ & 16 & 35 \\
Broberg-Morrey classification $(\%)^{\S}$ & III: 16 & II: 65 \\
& II: 84 & III: 15 \\
High-energy injury $(\%)$ & & IV: 20 \\
\hline
\end{tabular}

* Values are expressed as mean, with SD in parentheses; ${ }^{\dagger}$ occupation involving at least $15-\mathrm{kg}$ lifting; ${ }^{*}$ sports/recreation involving upperextremity weightbearing (ie, golf, basketball, volleyball); ${ }^{\S} \mathrm{I}=$ displaced $<2 \mathrm{~mm}$; II $=$ displaced $>2 \mathrm{~mm}$, involving $>30 \%$ of head; III = comminuted; IV = associated with dislocation; "fall from $>2$ feet $(61 \mathrm{~cm})$, fall during sport participation, motor vehicle collision; "unpaired t-test; "Fisher's exact test; **Mantel-Haenszel chi-square test; CESD = Center for Epidemiologic Studies Depression Scale.

was elevated to address associated injuries, if necessary $(\mathrm{n}=$ 2). The radial head was exposed through either a Kocher or a common extensor tendon splitting approach. Fractures were fixed with 1.5- to 3.0-mm-headed screws $(n=47)$ (Synthes, Mississauga, ON, Canada) or 3.2-mm headless screws ( $\mathrm{n}=3$ ) (Acutrak ${ }^{\mathrm{R}}$; Acumed, Hillsboro, OR, USA), except for one patient who had more than three radial head fragments, which were treated with a locking plate $\left(\right.$ Evolve $^{\circledR}$; Wright Medical Technology, Inc, Arlington TN, USA). Associated injuries of the lateral and medial collateral ligaments, olecranon, and coronoid were stabilized as necessary.

Postoperative rehabilitation protocols were individualized based on the associated injuries. Within 2 to 7 days postoperatively, all patients initiated active motion, supervised by a therapist. The protocols were modified according to the integrity of the collateral ligaments. For elbows requiring protection of the lateral collateral ligament, the forearm was maintained in a pronated position when the elbow was extended and active forearm rotation was only permitted with the elbow flexed greater than $90^{\circ}$ [12]. For elbows requiring protection of the medial collateral ligament, the forearm was maintained in a supinated position when the elbow was extended and active forearm rotation was only permitted with the elbow flexed greater than $90^{\circ}$ [34]. For elbows requiring protection of both the medial and lateral collateral ligaments, the forearm was maintained in a neutral position when the elbow was extended and active forearm rotation was only permitted with the elbow flexed greater than $90^{\circ}$. An overhead protocol was used in selected cases when necessary to manage more severe instability [36]. This technique uses gravity to add a compressive force to the elbow by performing exercises supine. As stability improved, static progressive extension splinting was employed to increase extension. Strengthening commenced between 6 and 12 weeks postoperatively when fracture healing was judged to be sufficient, typically when the fracture line relative to the radial neck was indistinct on followup radiographs.

The primary outcome measure, patient-reported pain and disability at a minimum of 1 year postoperatively, was quantified using the Patient-rated Elbow Evaluation (PREE) [27]. The PREE is a patient-based, validated, functional outcomes instrument. The main strengths of this instrument are the specific focus on function as influenced by elbow pathology and the values derived from the perspective of the patients. It does not include objective measures such as ROM or specific symptoms, except pain. Scores range from 0 to 100 , with lower scores representing lower pain and disability.

Radiographic outcome included an assessment of posttraumatic arthritis, fracture reduction, union, and heterotopic ossification. A clearly defined technique was employed using digital images only for the final followup radiographs (InteleViewer ${ }^{\mathrm{TM}}$; Intelerad Medical Systems Inc, Montreal, Quebec, Canada); initial radiographs were a mixture of hard copy films and digital images. The 
radiograph was enlarged on the viewing monitor so that the elbow maximally occupied the viewing screen. Measurements were achieved using the digital imaging measurement tools (InteleViewer ${ }^{\mathrm{TM}}$ ). Posttraumatic arthritis was graded on radiographs during the final followup visit according to the classification of Broberg and Morrey [3]: Grade 0, normal; Grade 1, slight joint space narrowing and minimal osteophyte formation; Grade 2, moderate joint space narrowing and moderate osteophyte formation; and Grade 3, severe degenerative change with gross destruction of the joint. Capitellar abnormality was defined as subtle irregularity of the subchondral bone, osteopenia, or cysts without joint space narrowing or osteophytes and therefore arthrosis not sufficient to warrant the diagnosis of Grade 1 radiographic arthritis. In comparison to immediate postoperative radiographs, loss of reduction was defined as any change involving greater than 1-mm articular step or gap or greater than $10^{\circ}$ angulation. Heterotopic ossification was measured according to the classification of Hastings and Graham [19]: Grade 1, without functional limitation; Grade 2, subtotal functional limitation $(\mathrm{A}=$ flexion arc deficit, $\mathrm{B}=$ forearm rotation deficit; $\mathrm{C}=$ both flexion arc and rotation deficit); and Grade 3, complete ankylosis. Postoperative complications were recorded, considering secondary or repeat surgery separately.

We performed statistical analyses using SAS ${ }^{\mathbb{R}}$ Version 9.3 (SAS Institute Inc, Cary, NC, USA), setting a level of significance at $p$ values of 0.05 or less. Descriptive statistics were performed to describe the means and SDs of continuous outcome variables and frequency for discrete variables. Univariate analysis comparing groups was performed using unpaired t-tests for continuous variables. Univariate analyses for discrete dependent variables were performed using Fisher's exact test. The Mantel-Haenszel chi-square test was used to compare ordinal data.
To show a 10-point difference between PREE scores (considered as a continuous variable) with a common SD of 10 points, comparative samples of 16 subjects would be necessary to demonstrate $80 \%$ power.

\section{Results}

With the numbers available, no differences between the simple and complex groups were noted in mean PREE scores ( 8 versus 15 , respectively; $p=0.13$ ) (Table 2 ).

With the numbers available, no differences were observed between the simple and complex groups in terms of radiographic degenerative radiocapitellar arthritis (32\% versus $40 \%$, respectively; $\mathrm{p}=0.76$ ) (Fig. 3), capitellar abnormality (61\% versus $60 \%$, respectively; $\mathrm{p}=1.0$ ) (Fig. 4 ), or major complication rate (collapse with prominent hardware, disabling elbow stiffness, or postoperative joint subluxation; $13 \%$ versus $25 \%$, respectively; $p=0.29$ ) (Table 2). However, capsular release rates in the simple and complex groups approached a statistical difference ( $3 \%$ versus $20 \%$, respectively; $\mathrm{p}=0.07$ ). Analysis of the capsular release cases suggested that capsular release may be more frequently observed when three or more fragments were present, but with the numbers available, we could not confirm this finding $(\mathrm{p}=0.08)$ (Table 3$)$.

\section{Discussion}

Radial head fractures can occur in isolation or in association with elbow and forearm injuries. The evidence supporting ORIF for repairable radial head fractures is inconclusive. We therefore compared patient-reported pain

Table 2. Outcomes (by group)

\begin{tabular}{|c|c|c|c|}
\hline Variable & $\begin{array}{l}\text { Simple group (isolated } \\
\text { radial head fractures) }(\mathrm{n}=31)\end{array}$ & $\begin{array}{l}\text { Complex group (complex fracture } \\
\text { patterns with radial head } \\
\text { fractures) }(\mathrm{n}=20)\end{array}$ & $\mathrm{p}$ value \\
\hline PREE (points)* & $8(13)$ & $15(20)$ & $0.13^{\#}$ \\
\hline Major complications $(\%)^{\dagger}$ & 13 & 25 & $0.29 * *$ \\
\hline Secondary capsular release $(\%)^{*}$ & 3 & 20 & $0.07 * *$ \\
\hline Prevalence of ulnotrochlear arthritis $(\%)$ & 13 & 25 & $0.29 * *$ \\
\hline Prevalence of radiocapitellar arthritis $(\%)^{\S}$ & 32 & 40 & $0.76^{* *}$ \\
\hline Prevalence of any capitellar abnormality $(\%)^{\|}$ & 61 & 60 & $1.0 * *$ \\
\hline Crepitus detected with motion $(\%)$ & 29 & 47 & $0.23 * *$ \\
\hline
\end{tabular}

* Values are expressed as mean, with SD in parentheses; ${ }^{\dagger}$ collapse with prominent hardware, disabling elbow stiffness (concomitant complex regional pain syndrome $[\mathrm{n}=1$; simple group], ulnar neuritis [ $\mathrm{n}=1$; complex group]), or posterolateral joint subluxation requiring external fixation revision ( $\mathrm{n}=1$; complex group); ${ }^{\ddagger}$ one patient underwent concomitant ulnar nerve transposition; ${ }^{\S}$ at least mild joint space narrowing and mild osteophyte formation (Broberg-Morrey Grade 1 posttraumatic arthritis of the elbow) compared to radiographs of the contralateral elbow;

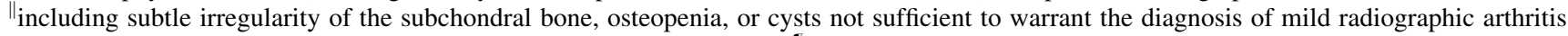
(any joint space narrowing, osteophyte formation compared bilaterally); "crepitus noted in the radiocapitellar joint and/or the ulnohumeral joint; \# unpaired t-test; **Fisher's exact test; PREE = Patient-rated Elbow Evaluation. 

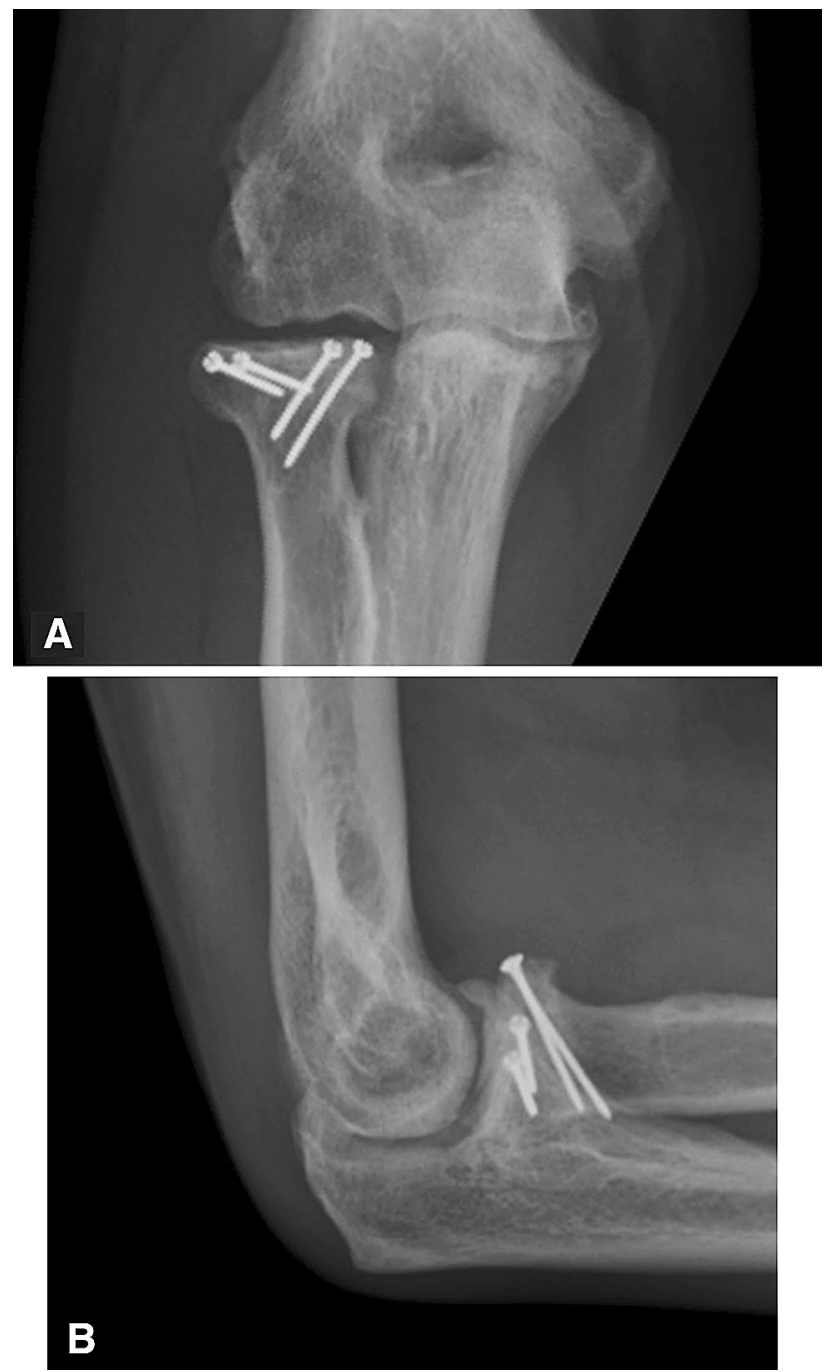

Fig. 3A-B (A) AP and (B) lateral radiographs of the patient shown in Figure 2 taken 4 years postoperatively after interim capsular release and coronoid hardware removal demonstrate progression of arthrosis despite maintained alignment. Note the capitellar osteopenia, subchondral bone irregularity, and ulnotrochlear degenerative changes.

and disability using a validated tool for the elbow (PREE), radiographic outcomes, complications, and reoperation rates in patients undergoing ORIF for isolated radial head fractures and for radial head fractures associated with other fractures or elbow dislocations.

This study has several limitations. The major limitation is the absence of a comparative group, specifically a cohort treated nonoperatively during the same period and a cohort treated with radial head arthroplasty. The outcome measurement tools are not specific to the radial head, and therefore, only the overall effect of the injury, including associated fractures and ligamentous injuries, can be demonstrated. As the complex group is heterogeneous with regard to both severity of injury and injured structures in
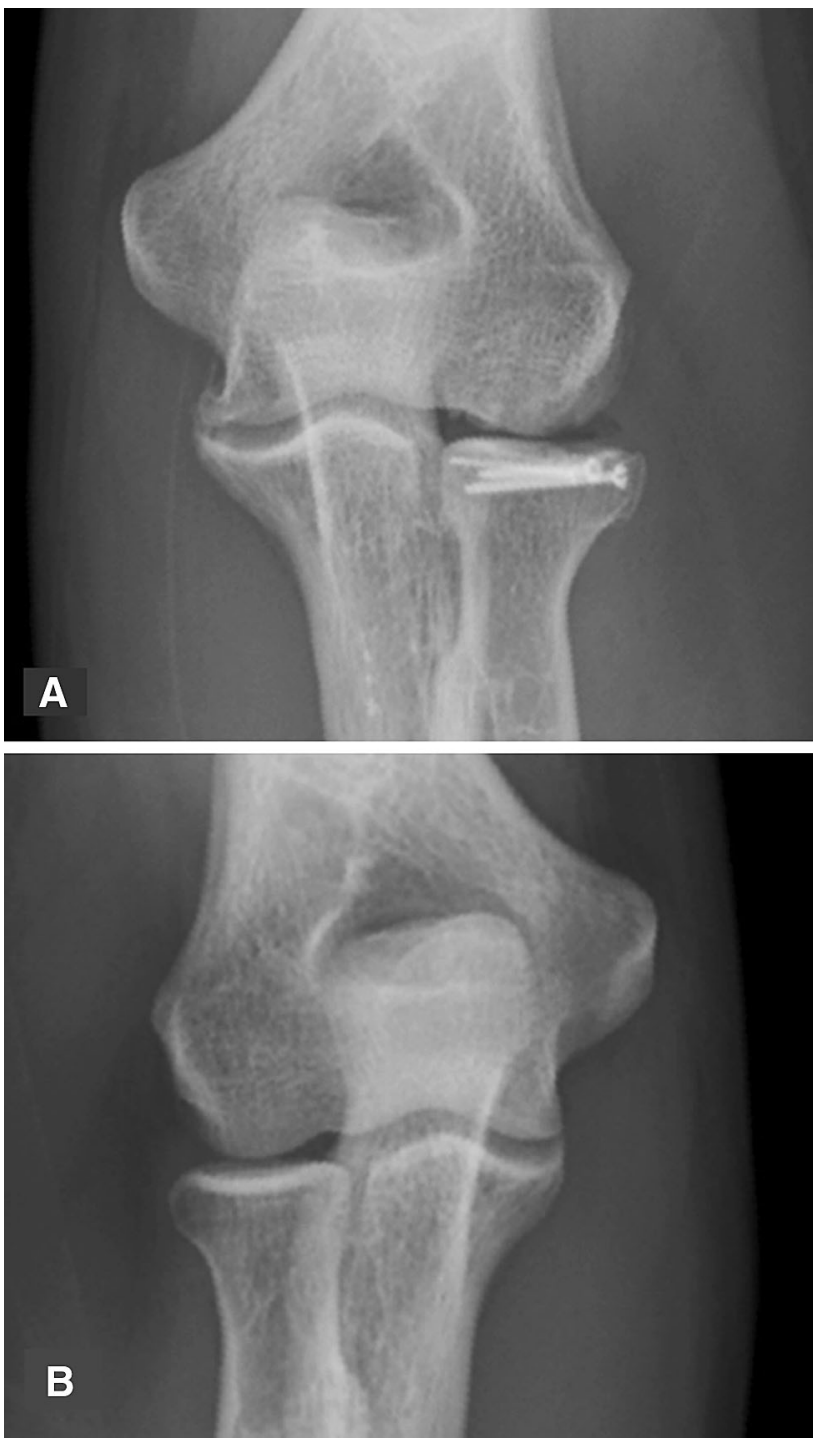

Fig. 4A-B (A) An example of capitellar abnormality at final followup is shown. Subtle irregularity in the subchondral bone and osteopenia are noted in this fracture, originally classified as an isolated radial head fracture. (B) The contralateral normal elbow is shown for comparison.

Table 3. Capsular release cases

\begin{tabular}{|c|c|c|c|}
\hline Variable & $\begin{array}{l}\text { Capsular } \\
\text { release } \\
(\mathrm{n}=5)\end{array}$ & $\begin{array}{l}\text { No capsular } \\
\text { release } \\
(n=46)\end{array}$ & $\mathrm{p}$ value* \\
\hline 3 or more fragments $(\%)$ & 60 & 20 & 0.08 \\
\hline $\begin{array}{l}\text { Complete articular } \\
\text { fractures }(\%)\end{array}$ & 40 & 13 & 0.17 \\
\hline High-energy injury $(\%)^{\dagger}$ & 81 & 60 & 0.64 \\
\hline $\begin{array}{l}\text { Prevalence of radiocapitellar } \\
\text { arthritis }(\%)\end{array}$ & 80 & 30 & 0.05 \\
\hline
\end{tabular}

* Fisher's exact test; ${ }^{\dagger}$ fall from a height greater than 2 feet $(61 \mathrm{~cm})$, fall during a sporting activity, or motor vehicle collision. 
addition to the radial head fracture, reliable extrapolation of the data to specific patients may be problematic.

Transfer bias (loss to followup) was a concern here as well, since only $63 \%$ of patients treated with ORIF for radial head fractures participated in this study; when such a large percentage of patients is lost to followup, one must surmise that the results risk overestimating the benefits of the treatments in question (in this case, surgical intervention). Patients lost to followup generally have poorer results compared with those who attend followup. However, the early complication rate and reoperation rate were likely less affected by the loss to followup. In addition, measurement bias is introduced by retrospective data collection of the descriptors of the initial injury and intraoperative findings. The use of radiographs likely introduced inaccuracy in injury classification. As this investigation was performed at a tertiary referral center, the sample studied may have included a higher proportion of complex injury patterns than those encountered in a general orthopaedic practice. Although the mean followup was 4 years in both groups, the minimum followup was only 1 year. One year is likely too early to accurately capture degenerative arthritis rates and therefore our study examines only early degenerative joint changes. The predominance of nonstatistically different comparisons between groups may represent Type II error; a larger sample size might have permitted demonstration of statistical difference between groups. A post hoc power analysis showed that, given the high pooled SD of 16 points for the PREE and the narrow difference between groups, this investigation was underpowered. To show a difference of 10 points in the PREE with the pooled SD observed in this study, 42 subjects per group would be required.

This cohort with radial head fractures treated with ORIF demonstrated little functional disability based on the PREE in both groups at latest followup. The low pain and disability were noted despite substantial rates of major complications in both the simple group (13\%) and the complex group (25\%), including collapse with prominent hardware, disabling elbow stiffness, and subluxation. Other investigations have shown favorable results from ORIF, with poorer outcomes noted with more complex injuries also $[13,25]$. This is in contrast to noncomminuted partial articular fractures that typically have achieved satisfactory outcomes after ORIF [33].

Subtle radiographic abnormalities of the affected capitellum were noted in the majority of patients in both groups, including irregularities in the subchondral bone, osteopenia, or cystic changes when compared to the contralateral side. While these findings do not meet the Broberg and Morrey criteria for arthritis, we believe these may represent early degenerative change. Further longterm followup is required. This observation may be a consequence of unrecognized chondral damage sustained at the time of initial injury, altered kinematics, and articular contact from residual ligamentous instability or subtle articular incongruity. Nonunion and proximal radioulnar synostosis have also been reported previously [33] but were not seen in the current series.

The potential complications from operative treatment should be weighed against the potential benefits. During the preoperative assessment, the complication rates merit discussion, particularly prominent hardware and stiffness, in an effort to reconcile patients' values with the potential pitfalls to ORIF of the radial head. The risks of disabling stiffness and subsequent capsular release appear to be greater in the complex group and therefore this discussion is particularly relevant in complex patterns of injury. The high rate of capsular release in the complex group was more commonly observed when three or more fragments were observed. Ring et al. [33] in a study of 56 patients noted poor results when radial head fractures with more than three fragments were treated with ORIF. Secondary radial head excision rates from $9 \%$ to $12 \%$ have been reported in series of patients treated nonoperatively [1,20]; radial head excision and arthroplasty were not utilized in the current study.

In summary, we found no differences in the short term between patients treated with ORIF for isolated radial head fractures and for radial head fractures in association with other elbow injuries. Loss to followup in this series precludes making strong statements regarding functional comparison between these groups. However, the substantial capsular release and complication rates in both groups should weigh into the preoperative discussion with patients before selecting ORIF for radial head fractures. This is particularly the case for radial head fractures in association with complex injury patterns with multiple radial head fragments where radial head arthroplasty may reduce the rate of secondary capsular release. Further research may help clarify this in the future.

Acknowledgments The authors acknowledge Chris Young BSc for his assistance in collecting data for this investigation.

\section{References}

1. Akesson T, Herbertsson P, Josefsson PO, Hasserius R, Besjakov $\mathrm{J}$, Karlsson MK. Primary nonoperative treatment of moderately displaced two-part fractures of the radial head. J Bone Joint Surg Am. 2006;88:1909-1914.

2. Boulas HJ, Morrey BF. Biomechanical evaluation of the elbow following radial head fracture: comparison of open reduction and internal fixation vs. excision, silastic replacement, and nonoperative management. Chir Main. 1998;17:314-320.

3. Broberg MA, Morrey BF. Results of delayed excision of the radial head after fracture. J Bone Joint Surg Am. 1986;68: 669-674. 
4. Broberg MA, Morrey BF. Results of treatment of fracture-dislocations of the elbow. Clin Orthop Relat Res. 1987;216:109-119.

5. Burkhart KJ, Mattyasovszky SG, Runkel M, Schwarz C, Kuchle R, Hessmann MH, Rommens PM, Lars MP. Mid- to long-term results after bipolar radial head arthroplasty. J Shoulder Elbow Surg. 2010;19:965-972.

6. Carstam N. Operative treatment of fractures of the head and neck of the radius. Acta Orthop Scand. 1950;19:502-526.

7. Chapman CB, Su BW, Sinicropi SM, Bruno R, Strauch RJ, Rosenwasser MP. Vitallium radial head prosthesis for acute and chronic elbow fractures and fracture-dislocations involving the radial head. J Shoulder Elbow Surg. 2006;15:463-473.

8. Cugola L, Vecchini L. [Use of a silastic prosthesis in traumatic and degenerative lesions of the radial head] [in Italian]. Clin Ortop. 1974;25:11-16.

9. Davidson PA, Moseley JB Jr, Tullos HS. Radial head fracture: a potentially complex injury. Clin Orthop Relat Res. 1993;297:224-230.

10. Dotzis A, Cochu G, Mabit C, Charissoux JL, Arnaud JP. Comminuted fractures of the radial head treated by the Judet floating radial head prosthesis. J Bone Joint Surg Br. 2006;88: 760-764.

11. Dubberley JH, Faber KJ, Macdermid JC, Patterson SD, King GJ. Outcome after open reduction and internal fixation of capitellar and trochlear fractures. J Bone Joint Surg Am. 2006;88:46-54.

12. Dunning CE, Zarzour ZD, Patterson SD, Johnson JA, King GJ. Muscle forces and pronation stabilize the lateral ligament deficient elbow. Clin Orthop Relat Res. 2001;388:118-124.

13. Esser RD, Davis S, Taavao T. Fractures of the radial head treated by internal fixation: late results in 26 cases. J Orthop Trauma. 1995;9:318-323.

14. Frosch KH, Knopp W, Dresing K, Langer C, Sturmer KM. [A bipolar radial head prosthesis after comminuted radial head fractures: Indications, treatment and outcome after 5 years] [in German]. Unfallchirurg. 2003;106:367-373.

15. Goldberg I, Peylan J, Yosipovitch Z. Late results of excision of the radial head for an isolated closed fracture. J Bone Joint Surg Am. 1986;68:675-679.

16. Grewal R, MacDermid JC, Faber KJ, Drosdowech DS, King GJ. Comminuted radial head fractures treated with a modular metallic radial head arthroplasty: study of outcomes. J Bone Joint Surg Am. 2006;88:2192-2200.

17. Harrington IJ, Sekyi-Otu A, Barrington TW, Evans DC, Tuli V. The functional outcome with metallic radial head implants in the treatment of unstable elbow fractures: a long-term review. J Trauma. 2001;50:46-52.

18. Harrington IJ, Tountas AA. Replacement of the radial head in the treatment of unstable elbow fractures. Injury. 1981;12:405-412.

19. Hastings $H$ 2nd, Graham TJ. The classification and treatment of heterotopic ossification about the elbow and forearm. Hand Clin. 1994;10:417-437.
20. Herbertsson P, Josefsson PO, Hasserius R, Karlsson C, Besjakov J, Karlsson M. Uncomplicated Mason Type-II and III fractures of the radial head and neck in adults: a long-term followup study. J Bone Joint Surg Am. 2004;86:569-574.

21. Ikeda M, Oka Y. Function after early radial head resection for fracture: a retrospective evaluation of 15 patients followed for 3-18 years. Acta Orthop Scand. 2000;71:191-194.

22. Ikeda M, Sugiyama K, Kang C, Takagaki T, Oka Y. Comminuted fractures of the radial head: comparison of resection and internal fixation. J Bone Joint Surg Am. 2005;87:76-84.

23. Ikeda M, Yamashina Y, Kamimoto M, Oka Y. Open reduction and internal fixation of comminuted fractures of the radial head using low-profile mini-plates. J Bone Joint Surg Br. 2003;85: 1040-1044.

24. Janssen RP, Vegter J. Resection of the radial head after mason type-III fractures of the elbow: followup at 16 to 30 years. J Bone Joint Surg Br. 1998;80:231-233.

25. Khalfayan EE, Culp RW, Alexander AH. Mason Type II radial head fractures: operative versus nonoperative treatment. J Orthop Trauma. 1992;6:283-289.

26. King GJ, Evans DC, Kellam JF. Open reduction and internal fixation of radial head fractures. J Orthop Trauma. 1991;5:21-28.

27. MacDermid JC. Outcome evaluation in patients with elbow pathology: issues in instrument development and evaluation. $J$ Hand Ther. 2001;14:105-114.

28. Odenheimer K, Harvey JP Jr. Internal fixation of fracture of the head of the radius. Two case reports. J Bone Joint Surg Am. 1979;61:785-787.

29. Osborne G, Cotterill P. Recurrent dislocation of the elbow. J Bone Joint Surg Br. 1966;48:340-346.

30. Radin EL, Riseborough EJ. Fractures of the radial head: a review of eighty-eight cases and analysis of the indications for excision of the radial head and non-operative treatment. J Bone Joint Surg Am. 1966;48:1055-1064.

31. Radloff LS. The CES-D scale: a self-report depression scale for research in the general population. App Psych Meas. 1977;1:385-401.

32. Ring D, Jupiter JB, Zilberfarb J. Posterior dislocation of the elbow with fractures of the radial head and coronoid. $J$ Bone Joint Surg Am. 2002;84:547-551.

33. Ring D, Quintero J, Jupiter JB. Open reduction and internal fixation of fractures of the radial head. J Bone Joint Surg Am. 2002;84:1811-1815.

34. Szekeres M, Chinchalkar SJ, King GJ. Optimizing elbow rehabilitation after instability. Hand Clin. 2008;24:27-38.

35. van Riet RP, Morrey BF. Documentation of associated injuries occurring with radial head fracture. Clin Orthop Relat Res. 2008;466:130-134.

36. Wolff AL, Hotchkiss RN. Lateral elbow instability: nonoperative, operative, and postoperative management. J Hand Ther. 2006;19: 238-243. 